\title{
Computational complexity of impact size estimation for spreading processes on networks
}

\section{Journal Article}

\section{Author(s):}

Laumanns, M.; Zenklusen, R.

Publication date:

2009

\section{Permanent link:}

https://doi.org/10.3929/ethz-b-000019034

\section{Rights / license:}

In Copyright - Non-Commercial Use Permitted

\section{Originally published in:}

The European Physical Journal B 71(4), https://doi.org/10.1140/epjb/e2009-00344-7 


\title{
Computational complexity of impact size estimation for spreading processes on networks
}

\author{
M. Laumanns ${ }^{\mathrm{a}}$ and R. Zenklusen \\ Institute for Operations Research, ETH Zurich, 8092 Zurich, Switzerland
}

Received 7 January 2009 / Received in final form 28 August 2009

Published online 17 October 2009 - (c) EDP Sciences, Società Italiana di Fisica, Springer-Verlag 2009

\begin{abstract}
Spreading processes on networks are often analyzed to understand how the outcome of the process (e.g. the number of affected nodes) depends on structural properties of the underlying network. Most available results are ensemble averages over certain interesting graph classes such as random graphs or graphs with a particular degree distributions. In this paper, we focus instead on determining the expected spreading size and the probability of large spreadings for a single (but arbitrary) given network and study the computational complexity of these problems using reductions from well-known network reliability problems. We show that computing both quantities exactly is intractable, but that the expected spreading size can be efficiently approximated with Monte Carlo sampling. When nodes are weighted to reflect their importance, the problem becomes as hard as the $s$ - $t$ reliability problem, which is not known to yield an efficient randomized approximation scheme up to now. Finally, we give a formal complexity-theoretic argument why there is most likely no randomized constant-factor approximation for the probability of large spreadings, even for the unweighted case. A hybrid Monte Carlo sampling algorithm is proposed that resorts to specialized $s$ - $t$ reliability algorithms for accurately estimating the infection probability of those nodes that are rarely affected by the spreading process.
\end{abstract}

PACS. 02.70.Tt Justifications or modifications of Monte Carlo methods - 64.60.aq Networks - 89.70.Eg Computational complexity - 89.75.-k Complex systems

\section{Introduction}

Abstract spreading processes, or reaction-diffusion processes, have become increasingly popular tools to study transmission and propagation of information (viruses, gossip, money, etc.) through populations of interacting entities (agents) [1]. Depending on the time scale, the propagation of information can be seen as a cascade of adverse effects or failures, such as financial defaults in credit networks, and the probability of a cascade affecting a large part of the population can therefore be seen as an indicator of the risk represented by the network's interaction structure [2].

In particular for the study of disease spreading, various models were introduced to describe the dynamics of the spreading process at different abstraction levels. Typically, the individual entities are in a certain state that can change over time, e.g., by interaction with other individuals. The main interest is then to predict, via computational or analytical methods, the dynamics or outcome of the spreading process, e.g., the number of affected individuals, and how the dynamics depends on the parameters

\footnotetext{
a e-mail: laumanns@ifor.math.ethz.ch
}

of the process. An important class of spreading models is based on differential equations, which can be handled efficiently and often yield analytical results for various quantities like the expected spreading size or the critical transmissibility at which a large part of the population gets affected [3]. The drawback is that homogeneity and perfect mixing within compartments is assumed.

As an obvious extension, a contact network can be introduced between the individuals. Such models are also called agent-based models and allow to reflect various mixing properties of the population as well as different infection rates between each pair of individuals. As pointed out already by Grassberger [4], SIR-type disease spreading models can be formulated as bond percolation problems, which allows to eliminate the time dependency of the spreading process. The spreading process can then be described as follows. Let $G=(V, E)$ be a directed network where for every arc $e=(v, w) \in E$ a spreading probability $p(e)$ is given corresponding to the probability that the disease will spread from node $v$ to node $w$ if $v$ is infected. Let $S \subseteq V$ a set of initially infected nodes. A possible outcome of this spreading process can be simulated by flipping a biased coin for every $\operatorname{arc}(v, w) \in E$ to determine whether it is an active arc meaning that the disease will spread 
from node $v$ to node $w$ if $v$ gets infected. Let $E^{\prime}$ be the set of active arcs. A particular node $v \in V$ gets infected if there is a path in $\left(V, E^{\prime}\right)$ from at least one node of $S$ to $v$. We assume w.l.o.g. that the set of initially infected nodes $S$ contains exactly one node $s$. The more general case with $|S|>1$ can easily be reduced to this case by introducing a new node $s$ which is infected and transmits the disease with probability one to all nodes of $S$. We call the triple $(V, E, p)$ a reliability network and the quadruple $(V, E, p, s)$ a spreading network. For a spreading network $G=(V, E, p, s)$ we denote by $I_{G}$ the random element containing all the nodes reached by the disease $\left(I_{G}\right.$ is thus a random subset of $V$ ).

This model can easily be extended to undirected or mixed networks, where an active undirected edge simply transmits the disease in both directions. Ball [5] showed that replacing every undirected edge by two oppositely directed edges with the same spreading probability as the original undirected edge does not change the model, i.e., if $G$ is the original spreading network and $G^{\prime}$ the one obtained by replacing the directed edges as described above then we have that $I_{G}$ and $I_{G^{\prime}}$ have the same distribution. The case of an undirected underlying network is therefore a special case of the directed version. As a natural extension, we can associate with every node $v \in V$ an integer weight $w(v) \in\{0,1,2, \ldots\}$ representing the impact of the spreading when reaching node $v$ and denote the weight of any subset of nodes $V^{\prime} \subseteq V$ by $w\left(V^{\prime}\right)=\sum_{v \in V^{\prime}} w(v)$.

Computing the probability that a specific node, all nodes, or, more generally, some given set of nodes $K \subseteq V$ will be covered by the spreading process are well studied problems known as the two-terminal, all-terminal and $K$-terminal reliability problems. All of them are known to be \#P-complete even on very restricted classes of networks [5-8]. In the context of spreading processes, however, one is often interested in more global properties instead of the probability that some given set of nodes will be reached by the process. We thus consider the following two questions:

1. Expected spreading size: what is the expected sum of weights of the nodes covered by the spreading process?

2. Probability of large spreadings: for a given $\alpha \in(0,1)$, what is the probability that the sum of weights of the nodes covered by the spreading is at least $\alpha w(V)$ ?

The above problems map to the unweighted case by choosing a weight equal to one for all nodes.

\section{Related work and our results}

There has been a lot of work (see e.g. [9-11] and references therein) on determining properties of spreading processes on ensembles of networks, which are characterized by specific distributions for the network data such as the degree distribution and the spreading probabilities. Typically, a generating function approach is used to derive analytical expressions for properties like the critical transmissibility or the expected spreading size [12], but usually the results are averages over the whole ensemble of networks considered and often rely on further assumptions like mean-field approximations or the non-existence of short cycles that are only valid in the infinite size limit. Nevertheless, the predictions obtained from such approximative models are in many cases remarkably good in the sense that they match with Monte Carlo simulations of the actual process $[11,12]$.

In a recent effort to determine thresholds for spreading processes on finite graphs rigorously without mean-field approximations, Draief et al. [13,14] have used probabilistic techniques to derive sufficient conditions for a spreading being small, i.e., affecting an asymptotically vanishing fraction with high probability. The analysis is based on the spectral radius of the adjacency matrix of the network, and the general result is that the spreading stays small if the ratio of cure to infection rates is larger than the spectral radius. The general result is then applied to different particular graph classes, partially with similar conditions for large spreadings. The example of the star network, for which the expected spreading size can be calculated exactly, shows that the general bound is close to the best possible. It is interesting to note that the analysis overestimates the infection of a given nodes via its neighbors using Boole's inequality. In fact, it is exactly the intricate dependency among the infection probabilities of the neighbors that makes exact computation of the individual infection probabilities (or $s$ - $t$ reliabilities) difficult. A further discussion about different techniques for analyzing spreading processes, the infinite population limit and the difficulty of short cycles for obtaining rigorous results is given in [15].

In this paper, we also follow a probabilistic approach, but we focus on computing the expected spreading size and the probability of large spreadings for a single (but arbitrary) given network. This is motivated by the fact that in practise one is often interested in very precise statements for particular network at hand, such as an actual airline network or a concrete water or gas distribution network [16]. In such examples, the actual structure might be very different from the ensemble averages of the considered graph class [17], which is often the case for complex engineered networks, especially when construction of the network was subject to a variety technological, physical, and financial constraints [18]. This setting can be seen as complementary to the one considered in $[13,14]$ and to the statistical physics approach, which focuses on ensemble averages and is instead able to make much more general statements about how properties of the spreading process depend on the network structure. As no generalpurpose methods besides Monte Carlo sampling seem to be available for computing or estimating the above quantities for a given networks up to now (except for simple special cases), we will study this issue by analysing the computational complexity (in the usual worst-case sense) of these tasks, by making use of existing results for reliability problems.

As computing infection probabilities for particular nodes is intractable in general, it is not very surprising 
that this holds also for the expected spreading size, which we show in this paper. One is therefore interested in efficient approximations. The most frequently used method for obtaining approximate solutions to the above problems is a direct Monte Carlo approach, where the spreading process is simulated multiple times and the quantity of interest is estimated by the sample average. This approach is known to be efficient only if the quantity to estimate is not too small. Thus, the difficult cases are the estimation of small expected spreading sizes and small probabilities of large spreadings, i.e., the estimation of rare events. When looking for estimation algorithms we are generally interested in $\epsilon-\delta$ approximations, which are algorithms returning a value accurate up to a relative error of $\epsilon$ with probability at least $1-\delta$. A fully polynomial randomized approximation scheme (FPRAS) is an $\epsilon-\delta$ approximation with a running time bounded by a polynomial in the input size and $1 / \epsilon$.

In Section 3, we show that the problem of computing the expected spreading size exactly is hard even when the underlying network is acyclic and unweighted. However, whereas the direct Monte Carlo approach is an FPRAS for the unweighted version, the estimation of the expected spreading size in the weighted version is computationally of the same difficulty as the $s$-t reliability problem, for which no FPRAS is known to date. In Section 4, we give a formal argument to show that most likely, there is no randomized constant-factor approximation for the probability of large spreading sizes even in the unweighted case. Finally, we propose a method for obtaining an $\epsilon-\delta$ approximation of the expected spreading size in the weighted version. The method is a hybrid of a direct Monte Carlo approach and existing $s$-t reliability estimators which are used as a black-box in our algorithm and whose usage will be limited since they are typically computationally expensive. Many existing $s$-t reliability estimators are designed for estimating very small reliabilities and may perform poorly when large reliabilities have to be estimated [19]. An important advantage of the proposed method is that the direct Monte-Carlo approach is only used for the estimation of relatively large reliabilities whereas the specialized $s$ - $t$ reliability estimators need only to estimate small reliabilities. Thus the proposed hybrid algorithm tries to exploit the complementary strength of both types of estimators. Although the algorithm is not guaranteed to be efficient, the performance is expected to be better than a direct Monte Carlo approach. Numerical results to demonstrate the behavior of the hybrid algorithm are given in Section 5 .

\section{Estimating the expected spreading size}

We start off by showing that exact computation of the expected spreading size is hard. More precisely, we show that the problem is $\# P$-complete, where $\# P$ denotes the complexity class of counting problems for which the number in question equals the number of accepting paths of a nondeterministic Turing machine. Typical \#P-complete

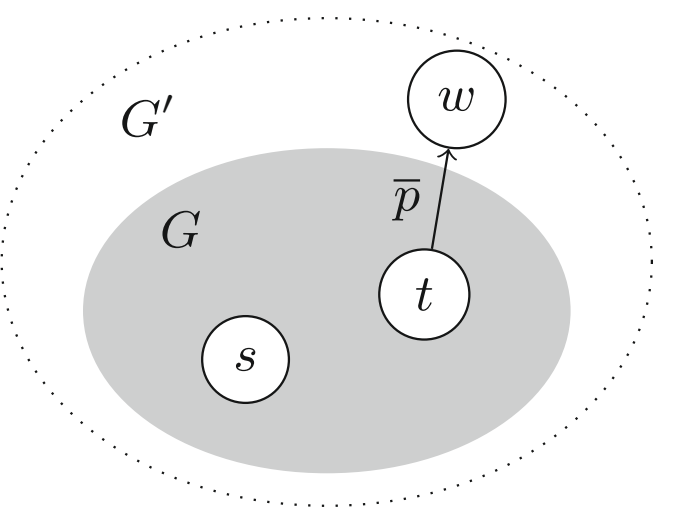

Fig. 1. Construction of the spreading network $G^{\prime}$ for the reduction from the $s$ - $t$ reliability problem in $G$ to the problem of computing the expected spreading size in $G^{\prime}$ used in the proof of Theorem 1.

problems include computing the permanent of a matrix [8] or the partition function of the Ising model on general graphs. For a discussion on the relationship of computational complexity of counting problems and statistical mechanics, we refer to [20]. In our case, the expected spreading size can be expressed as a weighted sum of $s$ - $t$ reliabilities, so the enumeration involved relates to the number of paths that the spreading can take to affect any given target node $t$.

Theorem 1. Computing the expected spreading size in the unweighted case is \#P-complete, even when restricted to acyclic networks and a uniform spreading probability $\bar{p} \in(0,1)$, i.e., $p(e)=\bar{p}$ for all $e \in E$.

Proof. To show that the problem is \#P-hard, we build a reduction from the $s$ - $t$ reliability problem with uniform failure probabilities, which is known to be \#Pcomplete [8]. Let $G=(V, E)$ be an acyclic network, $s \in V$ be the starting node of the spreading process, $\bar{p} \in(0,1)$ some fixed uniform spreading probability and $t \in V \backslash\{s\}$. Furthermore, let $G^{\prime}=\left(V^{\prime}, E^{\prime}\right)$ be the acyclic graph obtained from $G$ by adding a node $w$ and an arc from $t$ to $w$ (with spreading probability $\bar{p}$ ), see Figure 1 . By construction of $G^{\prime}$ we have $\mathrm{E}\left[\left|I_{G^{\prime}}\right|\right]=\mathrm{E}\left[\left|I_{G}\right|\right]+\mathrm{P}\left[w \in I_{G^{\prime}}\right]=$ $\mathrm{E}\left[\left|I_{G}\right|\right]+\bar{p} \mathrm{P}\left[t \in I_{G}\right]$. Thus, the $s$ - $t$ reliability $\mathrm{P}\left[t \in I_{G}\right]$ in $G$ can be determined as a function of $\mathrm{E}\left[\left|I_{G}\right|\right], \mathrm{E}\left[\left|I_{G^{\prime}}\right|\right]$ and $\bar{p}$, implying that computing the expected spreading size in an acyclic graph with uniform spreading probability is \#P-hard. Furthermore, the problem lies in \#P as it can be reduced to the $s$ - $t$ reliability problem by observing that $\mathrm{E}\left[\left|I_{G}\right|\right]$ can be expressed in terms of $s$ - $t$ reliabilities as $\mathrm{E}\left[\left|I_{G}\right|\right]=\sum_{v \in V} \mathrm{P}\left[v \in I_{G}\right]$.

Despite being \#P-complete, it is easy to obtain an FPRAS in the unweighted case just by applying a direct Monte Carlo approach since the expected spreading size is at least 1 as the node $s$ is always infected. This can be seen by applying the Generalized Zero-One Estimator Theorem [21], which shows that the direct Monte Carlo approach for estimating $\mathrm{E}\left[\left|I_{G}\right|\right]$ is an $\epsilon-\delta$ approximation if the number of iterations $N$ satisfies $N \geq 4(\mathrm{e}-2) \ln (2 / \delta)\left(|V| / \epsilon^{2}\right)$. The weighted case, however, 
is of the same difficulty as the $s$ - $t$ reliability problem, for which the existence of an FPRAS is still unresolved.

Theorem 2. Computing or estimating the expected spreading size in a weighted spreading network is of the same computational complexity as computing or estimating $s$-t reliabilities on the same underlying graph.

Proof. The $s$ - $t$ reliability of a reliability network $G=$ $(V, E, p)$ with $s, t \in V$ is the expected spreading size on the spreading network $G^{\prime}=(V, E, p, s, w)$ where the node $t$ has a weight equal to one and all other nodes have zero weight. On the other hand, the expected spreading size on a spreading network $G=(V, E, p, s, w)$ can be expressed as the weighted sum of $|V| s-t$ reliabilities as in the proof of Theorem 1 as $\mathrm{E}\left[\left|I_{G}\right|\right]=\sum_{v \in V} w(v) \mathrm{P}\left[v \in I_{G}\right]$. Thus, an $\epsilon-$ $\delta$ approximation for $\mathrm{E}\left[\left|I_{G}\right|\right]$ can be obtained by getting for every $v \in V$ an $\epsilon-\delta^{\prime}$ approximation $X_{v}$ of $\mathrm{P}\left[v \in I_{G}\right]$ with $\delta^{\prime}=\delta /|V|$ and estimating $\mathrm{E}\left[\left|I_{G}\right|\right]$ by $Y=\sum_{v \in V} w(v) X_{v}$. Hence, $Y$ is an $\epsilon$-approximation of $\mathrm{E}\left[\left|I_{G}\right|\right]$ if $X_{v}$ is an $\epsilon$ approximation of $\mathrm{P}\left[v \in I_{G}\right]$ for all $v \in V$, which happens with probability at least $1-|V| \delta^{\prime}=1-\delta$ (this error bound is even valid when the $s$ - $t$ estimators $\left\{X_{v} \mid v \in V\right\}$ are not independent).

\section{Estimating the probability of large spreadings}

Let $\alpha \in(0,1)$ be the threshold at which an outcome of a spreading process is considered as large; we call this an $\alpha$-spreading. The main idea in this section is to reduce the $K$-terminal reliability problem to the problem of estimating the probability of large spreading sizes. For this reduction we need the following simple observation.

Lemma 1. For every weighted spreading network $G=$ $(V, E, p, s, w)$ with positive integer weights $w$ an unweighted spreading network $G^{\prime}=\left(V^{\prime}, E^{\prime}, p^{\prime}, s^{\prime}\right)$ of size $\left|V^{\prime}\right|=w(V)$ and $\left|E^{\prime}\right|=|E|+w(V)-|V|$ can be constructed in $\mathcal{O}(|E|+w(V))$ time such that $w\left(I_{G}\right)$ and $\left|I_{G^{\prime}}\right|$ have the same distribution.

Proof. $G^{\prime}$ can be constructed on the base of $G$ by adding for every node $v \in V$ a set of $w(v)-1$ additional nodes and $\operatorname{arcs}$ from $v$ to the added nodes that are active with probability one. To verify that the distributions of $w\left(I_{G}\right)$ and $\left|I_{G^{\prime}}\right|$ are equal, one can observe the following correspondence between the random outcomes of the two spreading processes. Whenever a node $v \in V$, which contributes a value of $w(v)$ to the weighted spreading spreading size, is in $I_{G}$, its corresponding node $v^{\prime} \in V^{\prime}$ is in $I_{G^{\prime}}$ in the unweighted network, plus the $w(V)-1$ additional nodes that are connected to $v^{\prime}$ as their arcs are active with probability one. Conversely, any additional node can only be in $I_{G^{\prime}}$ if the node $v^{\prime}$ it is connected to is also in $I_{G^{\prime}}$, which corresponds to the event that its associated original node $v \in V$ is in $I_{G}$.

It is natural to expect that finding the probability of large spreadings should not be easier than finding the expected spreading size because by solving (respectively

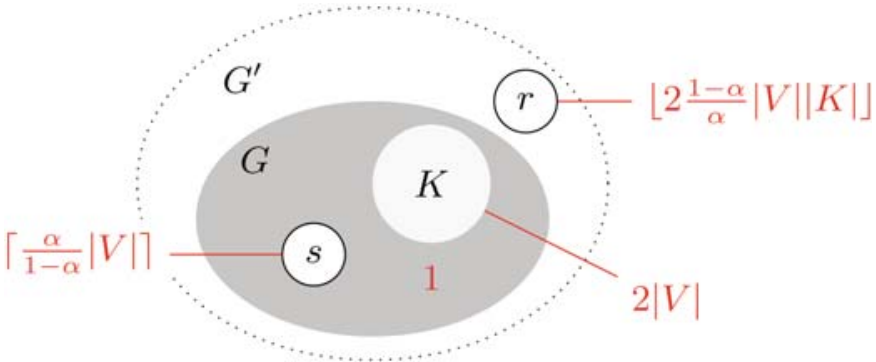

Fig. 2. (Color online) Construction of the weighted spreading network $G^{\prime}$ to which the $K$-terminal reliability problem is reduced in the proof of Theorem 3 .

approximating) the probability of large spreadings for different values of $\alpha$, we could determine (respectively estimate) the whole distribution of $\left|I_{G}\right|$ and not only its expected value. As usual, the direct Monte Carlo approach is an FPRAS when the probability of an $\alpha$-spreading is bounded by the reciprocal of a polynomial in the input size, but the following theorem shows that it is hard to approximate $\alpha$-spreading probabilities in general and implies that, unless $N P \subseteq B P P$, there is no randomized constantfactor approximation for this problem. Here, NP stands for the complexity class of decision problems solvable by a nondeterministic Turing machine in polynomial time (i.e., a given solution can be verified in polynomial time), and BPP (referring to Bounded-error, Probabilistic, Polynomial time) for the class of decision problems solvable by a probabilistic Turing machine in polynomial time with an error probability of at most $1 / 3$ for all instances.

Theorem 3. Unless $P=N P$, there is no constantfactor approximation for estimating the probability of $\alpha$-spreadings for any fixed $\alpha \in(0,1)$, even when the underlying network is unweighted.

Proof. Let $G=(V, E, p)$ be a reliability network, $s \in V$ and $K \subseteq V \backslash\{s\}$. We begin by reducing the $K$-terminal reliability problem on $G$, which asks to determine the probability that all nodes in $K$ can be reached from $s$ after the edge failures, to the problem of determining the probability of an $\alpha$-spreading in a weighted spreading network $G^{\prime}$ with positive integer weights. The network $G^{\prime}$ is obtained from $G$ by adding an additional isolated node $r$ with a weight of $\left\lfloor 2 \frac{1-\alpha}{\alpha}|K||V|\right\rfloor$. Furthermore, we assign a weight of $\left\lceil\frac{\alpha}{1-\alpha}|V|\right\rceil$ to the node $s$, a weight of $2|V|$ to each node in $K$, while other nodes have unit weight (see Fig. 2). We now have to check that a spreading in $G^{\prime}$ is an $\alpha$-spreading if and only if the spreading reaches all nodes in $K$. We can upper bound the weight of $G^{\prime}$ as

$$
\begin{aligned}
w\left(G^{\prime}\right) & =w(s)+w(V \backslash(K \cup\{s\}))+w(r)+w(K) \\
& =\left\lceil\frac{\alpha}{1-\alpha}|V|\right]+|V|-|K|-1 \\
& +\left\lfloor 2 \frac{1-\alpha}{\alpha}|V||K||+2| V|| K \mid\right. \\
& \leq \frac{1}{1-\alpha}|V|+\frac{2}{\alpha}|V||K|
\end{aligned}
$$


so hitting the set $K$ is sufficient for having an $\alpha$-spreading. A lower bound on $w\left(G^{\prime}\right)$ is given by

$$
\begin{aligned}
w\left(G^{\prime}\right) & =w(s)+w(V \backslash(K \cup\{s\}))+w(r)+w(K) \\
& =\left\lceil\frac{\alpha}{1-\alpha}|V|\right]+|V|-|K|-1 \\
& \left.+\left|2 \frac{1-\alpha}{\alpha}\right| V|| K \mid\right]+2|V||K| \\
& \geq \frac{1}{1-\alpha}|V|-|K|-2+\frac{2}{\alpha}|V||K|
\end{aligned}
$$

which together with the observation that

$$
w\left(G^{\prime}\right)-2|V|<w(s)+w(K)-2|V|+|V|-2<\alpha w\left(G^{\prime}\right)
$$

shows that hitting $K$ is also necessary to have an $\alpha$ spreading. Thus, the probability of an $\alpha$-spreading in $G^{\prime}$ is exactly the $K$-terminal reliability in $G$. Furthermore, the input size of $G^{\prime}$ is still bounded by a polynomial of the input size of $G$. Lemma 1 implies that the role of $G^{\prime}$ can be replaced by an unweighted spreading network with input size being polynomial in the size of $G$. Finally, since there is no constant-factor approximation for $K$-terminal reliability unless $P=N P[5]$, the theorem follows.

\section{A hybrid estimation algorithm}

We propose a simple hybrid algorithm for estimating the expected spreading size, which is based on the fact that an $\epsilon-\delta$ approximation of $\mathrm{E}\left[\left|I_{G}\right|\right]$ can be obtained by $\epsilon-\delta^{\prime}$ approximations of the $s-v$ reliabilities for all nodes $v \in V$ as shown in the proof of Theorem 2 . We can therefore profit from a large set of known algorithms for computing, approximating or bounding $s$ - $t$ reliability on different types of graphs [22-29].

The key idea is that we do not have to estimate the $s-t$ reliabilities separately for all nodes in $V$. As usual, the $s-v$ reliabilities that are not very small can easily be estimated by a direct Monte Carlo algorithm. This can be done by applying a direct Monte Carlo approach for some fixed number of iterations and determine for all nodes the fraction of outcomes in which they were reached by the spreading process. For each node $v \in V$ for which the estimation of the $s-v$ reliability by the direct Monte Carlo approach is not an $\epsilon-\delta^{\prime}$ estimation we estimate the $s-v$ reliability by applying one of the known $s$ - $t$ reliability estimation algorithms. Thus, the specialized algorithm is only applied for the estimation of small $s$ - $t$ reliabilities, which is the problem setting for which those algorithms are typically designed. To determine whether for some given node $v$, the estimated $s-v$ reliability is an $\epsilon-\delta^{\prime}$ approximation we used the criterion presented in the Stopping Rule Algorithm in [21].

To demonstrate the behavior of the hybrid estimation algorithm we give computational results on a directed acyclic Delaunay graph. An instance is characterized by two parameters, the number of nodes $n$ and a uniform arc

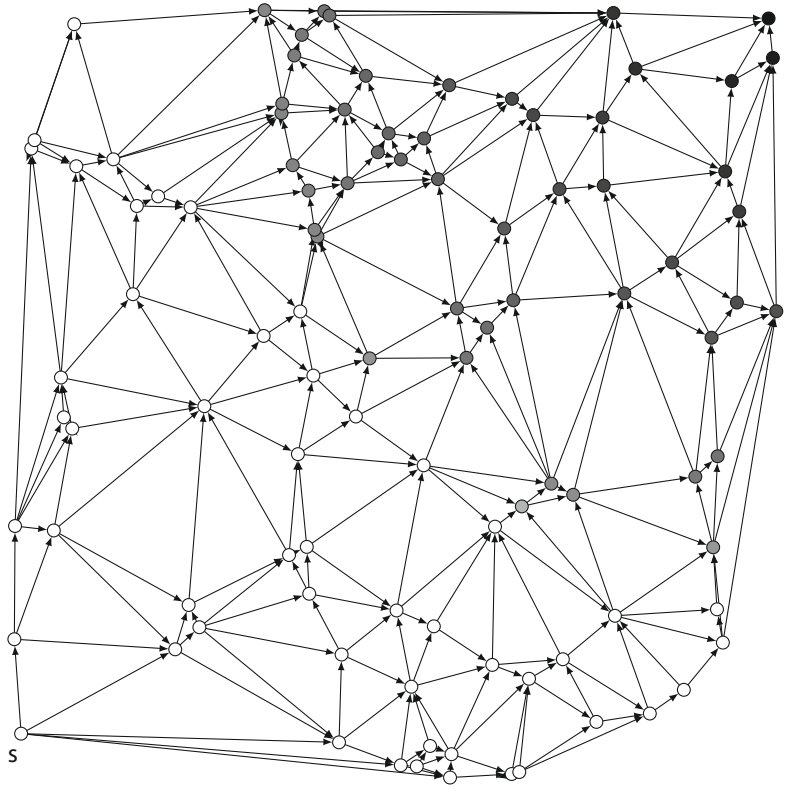

Fig. 3. Instance of a directed acyclic Delaunay graph used as a test case. The shading of the nodes indicates their weights.

spreading probability $\bar{p}$, and created as follows. We distribute $n$ nodes uniformly at random in the unit square and consider the undirected graph given by a Delaunay triangulation of these points. The initially infected node $s$ is chosen as the node closest to the origin. The edges are then oriented towards the node with the maximum Euclidean distance to $s$, which we call $t$. Thus, an (undirected) arc $\{v, w\}$ is oriented as $(v, w)$ if the vector from $v$ to $w$ and the one from $s$ to $t$ have a non-negative scalar product, otherwise we take the orientation $(w, v)$. We then equip all nodes with a weight that decreases exponentially with the distance from $t$. Figure 3 shows the generated test instance with the node weights indicated in different gray scales, from smallest (white) to largest (black) weight. We chose $n=100$ and $\bar{p}=0.1$.

As a Delaunay graph is planar, it allows a nice twodimensional illustration of the progression of the estimation accuracies for the individual nodes, as shown later in Figure 5. Moreover, the degree distribution is more heterogeneous compared to a regular lattice, which makes it more interesting, while the average path lengths are not too small. In other words, we chose an instance that should be difficult for simple Monte Carlo sampling and good to visualize.

We start the algorithm with target confidence $1-\delta$ with $\delta=0.001$ and target approximation error $\epsilon=0.01$. Figure 4 shows how the estimated weighted spreading size develops over time for the direct Monte Carlo sampling, which is applied in the first phase. It is noticeable that even after a large number of iterations, the Monte Carlo estimator fluctuates considerably, and the guaranteed accuracy of one percent is only reached after about $8 \times 10^{9}$ iterations (almost $10^{4} \mathrm{~s}$ of execution time). In comparison, we switch to the second phase and apply a dedicated $s$ - $t$ reliability approximation algorithm after 


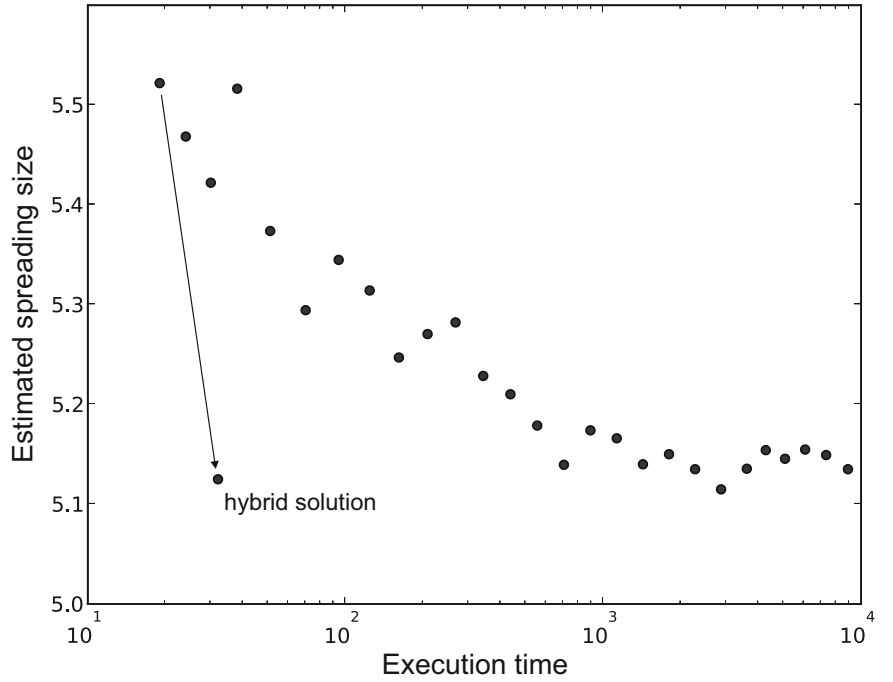

Fig. 4. Results of a sample run showing how the estimated spreading size develops over time.

$2 \times 10^{7}$ interactions, which corresponds to the first data point in Figure 4. At this point, the accuracy of the individual $s$ - $t$ reliabilities from the Monte Carlo sampling is as displayed in Figure 5, where the gray scale indicates the accuracy, and nodes beyond the dotted boundary are still not within the one percent accuracy range (for the adjusted confidence $1-\delta^{\prime}$ with $\left.\delta^{\prime}=\delta / n=10^{-5}\right)$. The final $\epsilon-\delta$ approximation after phase 2 is then obtained much faster, as indicated in Figure 4, which shows the benefit of the hybrid approach using targeted importance sampling.

\section{Conclusions}

The complexity of determining the expected final size of a spreading process and the probability of large spreadings in networks with unweighted and weighted nodes was analyzed. All of the considered problems are difficult when an exact solution has to be found. However, when we are interested in efficient approximations, the problems can be divided into three groups:

1. Problems for which an FPRAS is known (expected spreading size in the unweighted case).

2. Problems for which no FPRAS is known but we neither have an argument that it is hard to find one (expected spreading size in a weighted network), and

3. Problems for which no FPRAS is known, and we have an argument showing that it is hard to find one (probability of large spreadings in the weighted and unweighted case).

It would be of particular interest to close the gap for the problem of estimating the expected spreading size in a weighted network, that is, either to find an FPRAS or to prove that this problem is hard. As we have seen in Section 4 , this is equivalent to answering the same question for the $s$ - $t$ reliability problem. Another interesting direction would be to develop practically useful algorithms for

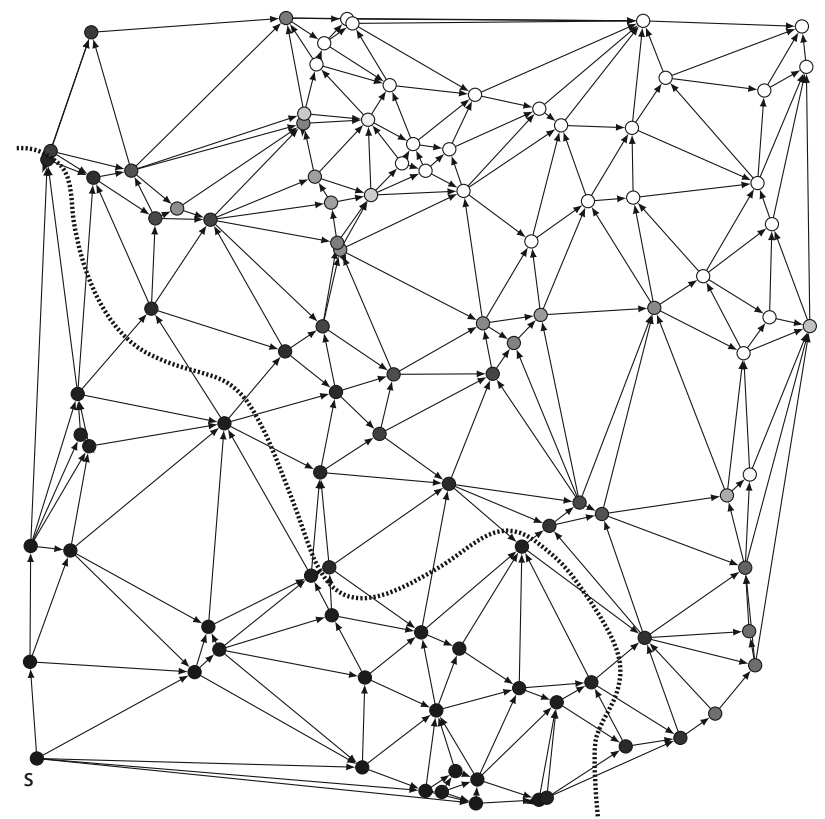

Fig. 5. Map of the accuracies obtained after $2 \times 10^{7}$ iterations of direct Monte Carlo sampling.

the different problems we studied for instances that cannot be solved efficiently by direct Monte Carlo simulation. The hybrid algorithm proposed here would be one option, although it is not guaranteed to be efficient.

The questions considered in this paper are mainly of computational nature, focusing only on the quantity of interest and not on how this number depends on structural properties of the network. The results are thus applicable to concrete networks whose link structure is completely known and relatively fixed compared to the time scale of the spreading process, and where it is important to determine the risk and impact of a spreading as precisely as possible for this particular network. This setting might also be relevant for the interaction networks of meta-population models [1], where the number of nodes (sub-populations) might be too small to justify the infinite size limit and exact computation on a single instance is the main interest.

\section{References}

1. A. Vespignani, Eur. Phys. J. B 64, 349 (2008)

2. J. Lorenz, S. Battiston, F. Schweitzer, Eur. J. Phys. B, present issue

3. J.D. Murray, Mathematical Biology (Springer, Berlin, 1989)

4. P. Grassberger, Mathematical Biosciences 63, 157 (1983)

5. M.O. Ball, Networks 10, 153 (1980)

6. J.S. Provan, SIAM Journal on Computing 15, 694 (1986)

7. J.S. Provan, M.O. Ball, SIAM Journal on Computing 12, $777(1983)$

8. L.G. Valiant, SIAM Journal on Computing 8, 410 (1979)

9. R. Pastor-Satorras, A. Vespignani, Phys. Rev. E 86, 3200 (2001) 
10. M.E.J. Newman, Phys. Rev. E 66, (2002)

11. Y. Moreno, R. Pastor-Satorras, A. Vespignani, Eur. Phys. J. B 26, 521 (2002)

12. J.C. Miller, Phys. Rev. E 76, 010101(R) (2007)

13. M. Draief, Phys. A 363, 120 (2006)

14. M. Draif, A. Ganesh, L. Massoulié, Annals of Applied Probability 18, 359 (2008)

15. J.C. Miller, J. Appl. Prob. 45, 498 (2008)

16. R. Carvalho, L. Buzna, F. Bono, E. Gutiérrez, W. Just, D. Arrowsmith, Phys. Rev. E 80, 016106 (2009)

17. D. Alderson, L. Li, Phys. Rev. E 75, (046102) (2007)

18. J.C. Doyle, D. Alderson, L. Li, S. Low, M. Roughan, S. Shalunov, R. Tanaka, W. Willinger, PNAS 102, 14497 (2005)

19. M. Laumanns, R. Zenklusen, in Proceedings of the European Conference on Complex Systems (ECCS), Dresden, Germany (2007)

20. R. Zecchina, Phys. A 302, 100 (2001)
21. P. Dagum, R. Karp, M. Luby, S. Ross, SIAM Journal on Computing 29, 1484 (2000)

22. W.W. Bein, J. Kamburowski, M.F.M. Stallmann, SIAM Journal on Computing 21, 1112 (1992)

23. M. Chari, T. Feo, J. Provan, Operations Research 14, 745 (1996)

24. C. Colbourn, L.D. Nel, T.B. Boffey, D.F. Yates, Annals of Operations Research 50, 173 (1994)

25. D.D. Harms, C.J. Colbourn, Networks 23, 289 (1993)

26. R. Karp, M. Luby, Journal of Complexity 1, 45 (1985)

27. M. Laumanns, R. Zenklusen, Estimation of small s-t reliabilities in acyclic networks, [arxiv.org/abs/0707.3231], (2007)

28. C. Lucet, J. Manouvrier, in Proceedings of 1st International Conference on Mathematical Methods in Reliability, Bucharest (1997)

29. A. Satyanarayana, R.K. Wood, SIAM Journal on Computing 14, (1985) 